\title{
Engineering of New Proteinoids and Proteinoid Nanoparticles of Narrow Size Distribution for Anti-fog Applications
}

Sason E*, Kolitz-Domb M*, Cohen S, Grinberg I and Margel S*

Department of Chemistry, the Institute of Nanotechnology and Advanced Materials, Bar Ilan University, Ramat Gan, Israel ${ }^{\#}$ These Authors Contributed Equally to this Study

\begin{abstract}
The "fog phenomenon" describes the formation of tiny droplets of water on different surfaces. In day-to-day life, fog affects the light transmission and damages the visibility of different surfaces, such as plastic packaging, lenses, mirrors and windshields. In this study, a new thin coating onto polypropylene films, made of proteionoids and proteinoid nanoparticles for fog prevention, is presented. The proteinoids and proteinoid nanoparticles were synthesized by thermal step-growth polymerization of amino acids and therefore are non-toxic, biodegradable and biocompatible. The anti-fogging ability of proteinoids and proteinoid nanoparticles was discussed in terms of wettability, surface chemistry and morphology, that were measured by contact angle and atomic force microscopy. The efficiency of the anti-fog coatings was also tested by hot and cold fog tests to examine the optical properties of the films under fog formation conditions. The obtained results revealed that the proteinoids and proteinoid nanoparticle coatings perform as a wetting enhancer, mainly due to the low water contact angle $\left(7-40^{\circ}\right)$, that can be attributed to the hydrophilic residues of the proteinoid. Furthermore, proteionoids and proteinoid nanoparticles improved the film roughness by smoothing the surface of films $(0.7-1.5 \mathrm{~nm})$. In fog tests, uncoated PP film display many small water drops on the surface that damaged the transparency of the film. In contrast, PP films coated with proteinoids or proteinoid nanoparticles formed a clear continuous thin layer of water on the surface. Additionally, the coating did not affect the clarity and haze of the films. Therefore, the coated films may be utilized in many applications, such as food packaging, agriculture and esthetic nylon wraps.
\end{abstract}

Keywords: Proteinoid nanoparticles; Thin coatings; Anti-fog coatings; Wettability; Surface roughness

\section{Introduction}

Proteinoids are polymers made from amino acids by thermal step-growth polymerization. Fox and coworkers discovered these polymers in the previous century while studying the origin of life [1-3]. Proteinoids can be used for many applications, such as drug delivery and diagnosis of diseases [4-7]. Proteinoids, made from natural amino acids, are non-toxic, non-immunogenic and biodegradable [7]. Following their synthesis, the proteinoids can self-assemble to form hollow nano or microparticles (NPs or MPs) due to the hydrophilic/ hydrophobic character of the different functional groups in the random polymer backbone [5]. When proteinoids are self-assembled in suitable conditions, the hydrophobic residues form a hydrophobic core in the inner side of the particle, in order to minimize their contact with water, while the hydrophilic groups remain on the outer shell of the particle and form hydrogen bonds with the water continuous phase. In addition, when the self-assembly procedure is performed in the presence of a suitable molecule, a proteinoid particle containing the molecule is formed [6]. Previous studies of proteinoids in our group presented dye and drug-filled proteinoid nanoparticles [4-7].

The manipulation of surface wettability through a combination of chemical and structural modifications has been of recent interest for a variety of applications such as anti-fog surfaces [8-10]. The "fog phenomenon" describes the condensation of water vapor to small drops that are dispersed on a solid surface. In our day-to-day life, fog is a problem in many technological products like plastic packaging, agricultural films and many optical devices, such as lenses, mirrors, windshields and visors. In all examples mentioned, fog causes a reduction in the light transmission, hence damaging the visibility of the products. In food packaging, the esthetics of the package is damaged, and the quality of the food may decrease to the point where it can no longer be sold due to the accumulated moisture $[8,11]$. In agricultural films, the fog droplets reduce light transmission and inhibit the growth of crops. Furthermore, the water droplets can cause the "lens effect", where sunlight is being focused, and harms the plant top leaves [11]. One way to avoid water condensation is to control the temperature and humidity of the environment. The other approach is to use antifog coatings on the surface of devices, which is preferable due to lower costs and lower energy consumption. The degree of hydrophilicity of surfaces, measured by water droplet contact angle, provides a measure for their anti-fogging ability. Generally, surfaces with a water contact angle degree of less than $40^{\circ}$ are often explored as anti-fog surfaces [10]. The main reason is that condensing water droplets on this type of surface can rapidly spread into a uniform and non-light-scattering water film. In this case, although condensation still occurs, the surface remains optically clear, without disruption of light transfer. Hydrophilic polymeric systems containing hydroxyl groups $(\mathrm{OH})$, amino groups $\left(\mathrm{NH}_{2}\right)$ and carboxylic groups $(\mathrm{COOH})$ are often utilized as anti-fog formulas. Another important property of anti-fog films is the roughness of the film surface. Fog will accumulate on rough surfaces, as the water droplets penetrate the holes in the surface and stay there [8]. The combination of both a smooth surface and a low water contact angle yields a good anti-fog film. However, the preparation of optical quality thin-film coatings with good coating characteristics and mechanical durability is still a great challenge [10].

${ }^{*}$ Corresponding author: Solomo Margel, Department of Chemistry, the Institute of Nanotechnology and Advanced Materials, Bar Ilan University, Ramat Gan 52900 , Israel, Tel: 97235318861; E-mail: shlomo.margel@biu.ac.il

Received: November 27, 2017; Accepted: December 06, 2017; Published: December 11, 2017

Citation: Sason E, Kolitz-Domb M, Cohen S, Grinberg I, Margel S (2017) Engineering of New Proteinoids and Proteinoid Nanoparticles of Narrow Size Distribution for Anti-fog Applications. J Nanomed Nanotechnol 8: 473. doi: 10.4172/2157-7439.1000473

Copyright: (C) 2017 Sason E, et al. This is an open-access article distributed unde the terms of the Creative Commons Attribution License, which permits unrestricted use, distribution, and reproduction in any medium, provided the original author and source are credited. 
In this study, we present new anti-fog coatings on polypropylene (PP) plastic films. The anti-fog coatings made from proteinoids or proteinoid particles, either hollow or encapsulating sorbitan monoleate (SMO). SMO is a small polar amphiphilic molecule, commonly used as an additive for food packaging and is approved for food contact by FDA, Figure 1 [11]. In recent studies, SMO was used in the compounding of linear low density polyethylene (LLDPE) and its anti-fog ability was investigated. In our study, SMO was used as an anti-fog additive, encapsulated within the proteinoid NPs, in order to achieve a good anti-fogging effect with the release of SMO molecules from the particle interior. The SMO-filled NPs were compared to hollow NPs to see the direct effect of the proteinoid itself as an anti-fog agent.

\section{Materials and Methods}

\section{Materials}

The following analytical-grade chemicals were purchased from commercial sources and were used without further purification: (L) glutamic acid (E), (L)phenylalanine (F), (L)isoleucine (I), surbitan monooleate (SMO), brilliant blue FCF, human serum albumin (HSA), Triton-x-100 and bovine plasma fibrinogen from Sigma (Rehovot, Israel); phosphate buffered saline (PBS), minimum essential medium Eagle's supplement (MEM), fetal bovine serum (FBS), glutamine, penicillin, streptomycin and mycoplasma detection kit from Biological Industries (Bet Haemek, Israel); cell cytotoxicity assay kit (LDH) from Roche (Switzerland); human osteosarcoma U-2 OS cell line from American Type Culture Collections (ATCC, VA,USA); PP films (air corona treated) of A4 size and $30 \mu \mathrm{m}$ average thickness from Dor Film Ltd., Israel. Standard glass microscope slides and primer (film former) A131-X from MICA corporation (CT, USA). Water was purified by passing deionized water through Elgastat Spectrum reverse osmosis system (Elga Ltd., High Wycombe, UK).

\section{Preparation and characterization of proteinoids by thermal step-growth polymerization}

Three amino acids were used to prepare a series of proteinoids: (L) glutamic acid (Glu, E), (L)phenylalanine (Phe, F) and (L)isoleucine (Ile, I) in different weight ratios, to give a total monomer weight of $5 \mathrm{~g}$, as specified in Table 1 . Each mixture of amino acids was heated in a heating mantle to $180^{\circ} \mathrm{C}$, under nitrogen. The mixture was kept at $180^{\circ} \mathrm{C}$ and mechanically stirred at $150 \mathrm{rpm}$ for $45 \mathrm{~min}$, to yield a brown glassy mass. When the mixture cooled to room temperature,

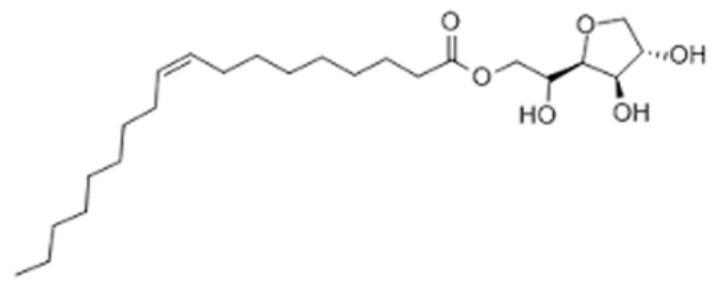

Figure 1: Chemical structure of sorbitan monoleate (SMO).

\begin{tabular}{|l|c|c|c|}
\hline \multirow{2}{*}{ Proteinoid } & \multicolumn{3}{|c|}{ Amino acid content $(\mathbf{g})^{\mathbf{a}}$} \\
\cline { 2 - 4 } & (L)Glu & (L)lle & (L)Phe \\
\hline $\mathrm{P}(\mathrm{EI})$ & 2.5 & 2.5 & - \\
\hline $\mathrm{P}(\mathrm{EIF})$ & 2.5 & 1.25 & 1.25 \\
\hline $\mathrm{P}(\mathrm{EF})$ & 2.5 & - & 2.5 \\
\hline
\end{tabular}

aTotal monomer weight is $5 \mathrm{~g}$

Table 1: Amino acid content of the different proteinoids. water $(15 \mathrm{~mL})$ was added to the crude product, and the mixture was stirred overnight. The solution was then intensively dialyzed through a cellulose membrane (500-1000 Da MWCO) against distilled water. The content of the dialysis tube was then lyophilized to obtain a yellowwhite proteinoid powder.

The molecular weights and polydispersity indices of the proteinoids were determined using Gel Permeation Chromatography (GPC) consisting of a Waters Spectra Series P100 isocratic HPLC pump with an ERMA ERC-7510 refractive index detector and a Rheodyne (Coatati, CA) injection valve with a $20 \mu \mathrm{L}$ loop (Waters, MA). The samples were eluted with super-pure HPLC water through a linear BioSep SEC-s-3000 column (Phenomenex) at a flow rate of $1 \mathrm{~mL} / \mathrm{min}$. The molecular weight was determined relative to poly(ethylene glycol) standards (Polymer Standards Service-USA, Silver Spring, MD, USA) with a molecular weight range of 100-450,000 Da, HSA (67 kDa), and bovine plasma fibrinogen $(340 \mathrm{kDa})$, using Clarity chromatography software.

The optical activities of the proteinoids were determined using a PE 343 polarimeter (PerkinElmer). The measurements were done in water, at $589 \mathrm{~nm}$ and $25^{\circ} \mathrm{C}$.

In order to determine the free carboxyl groups in the synthesized proteinoids, a titrimetric method was carried out [12]. Briefly, to a known quantity of dry proteinoid, a known excess of $0.05 \mathrm{~N} \mathrm{NaOH}$ was added, followed by the addition of $37 \%$ formaldehyde solution. The unreacted $\mathrm{NaOH}$ was back-titrated with standard $0.05 \mathrm{~N} \mathrm{HCl}$. A blank titration was also performed. In addition, human serum albumin (HSA) was titrated for comparison.

\section{Preparation of proteinoid nanoparticles by self-assembly}

Proteinoid particles were prepared by a self-assembly mechanism $[4-7,13]$. Briefly, $100 \mathrm{mg}$ of the dried proteinoid were added to $10 \mathrm{~mL}$ of $10^{-5} \mathrm{~N} \mathrm{NaCl}$ solution. The mixture was then heated to dissolution of the proteinoid, at $80^{\circ} \mathrm{C}$. Proteinoid particles were formed by self-assembly upon removal of the heating and slow cooling to room temperature.

Encapsulation of the anti-fogging agent sorbitan monooleate (SMO) was performed similarly. To the heated proteinoid mixture $(100 \mathrm{mg}$ in $9.8 \mathrm{~mL} \mathrm{NaCl} 10^{-5} \mathrm{~N}$, at $\left.80^{\circ} \mathrm{C}\right), \mathrm{SMO}(10 \%$ relative to the proteinoid, 10 $\mathrm{mg}$ in $200 \mu \mathrm{L}$ ) was added. The heating was stopped and the mixture was mechanically stirred at $250 \mathrm{rpm}$ and left to cool to room temperature in order to form proteinoid particles containing SMO.

The obtained particles were dialyzed through a cellulose membrane (500-1000 Da MWCO) against distilled water to wash-off excess reagents.

The hydrodynamic diameter and size distribution of the nanoparticles were measured at room temperature by a DLS analyzer Vasco 2, Particle Sizing System (Cordouan Technologies SAS, France).

In addition, the size and size distribution of the NPs were measured with a cryogenic transmission electron microscope (cryo-TEM). For this purpose, a small droplet of an aqueous dispersion of the NPs was placed on a perforated lasy carbon film supported on a TEM copper grid held by tweezers. The drop was blotted with a piece of filter paper, resulting in the formation of thin films of $100-300 \mathrm{~nm}$. The specimen was subsequently plunged into a reservoir of liquid ethane cooled by liquid nitrogen to ensure its vitrification (rapid freezing) and to prevent ice crystal formation. The vitrified specimen was transferred under liquid nitrogen and mounted on a cryogenic sample holder cooled to $-170^{\circ} \mathrm{C}$. All samples were observed under low-dose conditions. Vitrified 
samples were examined in an FEI T12 G2 Cryo-TEM operating at 120 $\mathrm{kV}$, equipped with a Gatan 626 cryo-holder system. The mean diameter was determined by measuring $200 \mathrm{NPs}$ using an image analysis software (AnalySIS Auto, Soft Imaging SystemGmbH, Germany).

Fourier transform infrared measurements were performed by the attenuated total reflectance (ATR) technique, using a Bruker ALPHAFourier transform infrared QuickSnap ${ }^{\text {Tw }}$ sampling module equipped with a Platinum attenuated total reflectance diamond module.

The thermal behavior of the proteinoids and proteinoid particles was determined using thermo gravimetric analysis (TGA) with a TGA/ DSC 1 STARe system (Mettler Toledo, Switzerland). The samples were heated at $25-700^{\circ} \mathrm{C}$ at a rate of $10^{\circ} \mathrm{C} / \mathrm{min}$ under nitrogen atmosphere.

\section{Preparation of the anti-fog coatings by spreading}

Polypropylene (PP) films were coated by spreading with a coating rod method. The PP was pre-treated by air corona to improve the adhesion of the coatings. A131-X, a typical adhesive aqueous solution of polyethyleneimine widely used as a commercial film former, was used in order to allow good adhesion between the anti-fog additives and substrate surface. First, a mixture of A131-X and proteinoid particles or crude proteinoid was prepared ( $5 \% \mathrm{w} / \mathrm{w}$ proteinoid to film former). Then, the mixture was spread onto the surface of the substrate by using Mayer rod (RK Print Coat Instruments Ltd., Litlington, Royston) $[14,15]$. Following this coating process, the substrate was dried in a heating oven at $80^{\circ} \mathrm{C}$ for a few minutes.

\section{Preparation of the anti-fog coatings by spray}

Glass slides were coated by a spray method. Briefly, a mixture of A131-X and crude proteinoid $\mathrm{P}(\mathrm{EI})$ was prepared (100 mg proteinoid

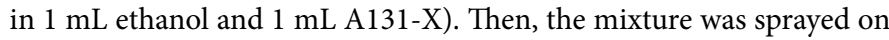
the surface of the glass by using a commercial spraying bottle. Following this coating process, the substrate was dried at room temperature for a few minutes.

\section{Characterization of the anti-fog coatings}

Surface topography analysis of the coated and uncoated PP were obtained using atomic force microscopy (AFM, NanoScope9, Bio FastScan, Bruker AXS, Santa Barbara, CA) with 1-3 N/m ScanAsyst-Air probe (Bruker, Santa Barbara, CA). Scans for each film were performed on an area of $3 \mu \mathrm{m} \times 3 \mu \mathrm{m}$ using the ScanAsyst mode with a $2 \mathrm{~Hz}$ scan rate. The height images were captured with 512 scans/line image resolution. AFM images were obtained in air at RT. Before analysis of the images, the second order "flatting" and first "planefit" functions were applied to each image. The analysis of the height images was done by using the NanoScope Analysis Software. The morphological changes of the films were determined by the root mean square roughness $(\mathrm{Rq})$ values averaged over three different regions on each film. The reported values are an average of at least 3 different points of four different films.

The sessile drop measurements (water contact angle) were done using a Goniometer (System OCA, model OCA20, Data Physics Instruments $\mathrm{GmbH}$, Filderstadt, Germany). Drops of $5 \mu \mathrm{L}$ distilled water were dropped on five different areas of each film and images were captured a few seconds after the deposition. The static water contact angle values were performed using Laplace-Young curve fitting. All of the measurements were done at $25^{\circ} \mathrm{C}$ and $60 \%$ moisture. Each result represents an average of 4 measurements with up to $5 \%$ standard deviation. Uncoated PP was used as a reference.

In addition, a solution of distilled water colored by brilliant blue
FCF was dropped on a PP film uncoated and coated with P(EI). The images clarifying the differences between the films were taken at several time periods using a digital camera (Canon EOS 550d).

Haze measurements were performed using Haze-Gard Plus 4752 model with ASTM D1003 standard (BYK-Gardner, Germany). Gloss measurements were performed using Micro-Gloss $45^{\circ} 4535$ model with ASTM D2457 standard (BYK-Gardner, Germany).

The anti-fog behavior of the films and glass was studied using a hot fog test and a cold fog test $[11,16]$, conducted as follows. An open $28 \mathrm{~mL}$ vial filled with $10 \mathrm{~mL}$ water was covered with a $5 \mathrm{~cm} \times 5 \mathrm{~cm}$ film, subsequently kept in a $60^{\circ} \mathrm{C}$ water bath or $4^{\circ} \mathrm{C}$ in refrigerator for $180 \mathrm{~min}$. Variations of the optical visibility of the films were observed and recorded at different time intervals. Ratings of A to D were used, where $\mathrm{D}$ denotes zero visibility with an opaque layer of small water droplets and A describes excellent optical visibility where a transparent continuous film of water is displayed.

The stability of the coatings was examined. Briefly, the coated films were put in a laboratory cupboard for 12 months. The films were taken at different time periods for hot fog test as described above.

\section{In vitro cytotoxicity of the proteinoids and proteinoid nanoparticles}

In vitro cytotoxicity of the proteinoids and proteinoid nanoparticles were tested by using human osteosarcoma U-2 OS cell lines. The cells are adherent to the used culture dishes. U-2 OS cells were grown in MEM with heat-inactivated FBS (10\%), penicillin (100 IU/mL), streptomycin $(100 \mu \mathrm{g} / \mathrm{mL})$ and (L)glutamine $(2 \mathrm{mM})$.

Cell cytotoxicity was measured by quantification of the release of cytoplasmic lactate dehydrogenase (LDH) into cell culture supernatants. LDH activity was assayed using the cytotoxicity detection kit according to the manufacturer's instructions [17]. Cells $\left(3 \times 10^{5}\right)$ were seeded and grown to $90-95 \%$ confluency in 24 -well plates before treatment with the proteinoids and proteinoid NPs. Untreated cells were included in all assays as negative controls. Cells treated with $1 \%$ Triton- $\mathrm{x}-100$ were used as positive control. Cells were exposed to a mixture containing maximal concentrations of proteinoid or NPs $(1 \mathrm{mg} / \mathrm{mL})$ in PBS and $1 \%$ Triton-x-100 in order to provoke possible interaction with the kit components. Cells were incubated with the proteinoids and proteinoid particles dispersed in PBS $(1 \mathrm{mg} / \mathrm{mL}$ and $0.1 \mathrm{mg} / \mathrm{mL})$ at $37^{\circ} \mathrm{C}$ in a humidified $5 \% \mathrm{CO}_{2}$ incubator and then checked for cellular cytotoxicity at intervals of $72 \mathrm{~h}$. The percentage of cell cytotoxicity was calculated using the formula shown in the manufacturer's protocol [17]. All samples were tested in tetraplicates.

XTT assay was performed to determine the viability of the human osteosarcoma U-2 OS cell lines after proteinoids and particles treatment. The assay is based on the ability of the mitochondrial succinate-terazolium reductase system to convert yellow tetrazolium salt XTT (sodium 3'-[1-(phenylaminocarbonyl)-3,4-tetrazolium]bis(4-methoxy-6-nitro)benzene sulfonic acid hydrate) to orange formazan dye [18]. The test denotes the percentage of the survived cells after toxic exposure. Cells were seeded in a 96-well plate at a density of $10^{4}$ cells/well in $100 \mu \mathrm{L}$ culture medium and grown in a humidified $5 \% \mathrm{CO}_{2}$ atmosphere at $37^{\circ} \mathrm{C}$. After $24 \mathrm{~h}$ at $37^{\circ} \mathrm{C}$, different volumes of the proteinoid and proteinoid particles dispersed in PBS were added to the cells, giving final concentrations of 1 and $0.1 \mathrm{mg} / \mathrm{mL}$ per well. After incubation for $72 \mathrm{~h}$ at $37^{\circ} \mathrm{C}, 50 \mu \mathrm{L}$ XTT solution was added to each well according to the kit manufacturer's instructions. Absorbance was read at $480 \mathrm{~nm}$. Cell viability was determined using the formula shown in 
Citation: Sason E, Kolitz-Domb M, Cohen S, Grinberg I, Margel S (2017) Engineering of New Proteinoids and Proteinoid Nanoparticles of Narrow Size Distribution for Anti-fog Applications. J Nanomed Nanotechnol 8: 473. doi: 10.4172/2157-7439.1000473

the manufacturer's protocol [18]. Reference wavelength used was 650 nm.

\section{Results and Discussion}

\section{Synthesis and characterization of the proteinoids}

Proteinoids were prepared by thermal condensation of different amino acids and characterized for their molecular weights, polydispersity, optical activity and free carboxyl group, as shown in Table 2. The synthesized proteinoids were of relatively high molecular weights, in the range of $57-68 \mathrm{kDa}$ with narrow polydispersity index (PDI) in the range of 1.04-1.07. This indicates that the simple thermal polymerization procedure used here provides relatively long polymer chains with a very narrow PDI, as already reported in previous studies of our group [4-7,19]. Furthermore, Table 2 shows that all proteinoids exhibit optical activity, although the monomers are known to racemize during the thermal process [20]. All proteinoids display approximately similar values of free carboxyl groups, between $85-97 \mathrm{mmol} / \mathrm{g}$. The proteinoids were compared to the natural protein albumin, which possess $56 \mathrm{mmol} / \mathrm{g}$ carboxylic groups. The amount of the carboxyl groups in the proteinoids is higher than the amount of carboxyl groups in albumin, due to the high percentage of glutamic acid in the proteinoid.
Polymers containing hydroxyl groups $(\mathrm{OH})$, amine groups $\left(\mathrm{NH}_{2}\right)$ and carboxyl groups $(\mathrm{COOH})$ are often utilized in anti-fog formulas [21]. Hence, the amount of the free carboxyl group in the proteinoids is very important.

\section{Characterization of proteinoid nanoparticles}

Proteinoid NPs were prepared by self-assembly and characterized for their size distribution and thermal properties (Table 3). The hollow $\mathrm{P}(\mathrm{EI}) \mathrm{NPs}$ produced a single population of hollow spherical particles in the range of $89.7 \pm 6 \mathrm{~nm}$ diameter as shown by the DLS histogram (Figure 2A) and $73.0 \pm 19 \mathrm{~nm}$ as shown by the cryo-TEM measurements (Figure 2C). SMO-filled P(EI) NPs were larger, containing a single population of a diameter of $166.1 \pm 20 \mathrm{~nm}$ according to the DLS measurements (Figure 2B) and $172.4 \pm 21 \mathrm{~nm}$ as measured by the cryoTEM (Figure 2D). $\mathrm{P}(\mathrm{EIF})$ and $\mathrm{P}(\mathrm{EF})$ yielded larger particles, either hollow or SMO-filled. Hence, $\mathrm{P}(\mathrm{EI})$ is preferable for further research.

Decomposition temperatures of the different proteinoids range between 290 and $315^{\circ} \mathrm{C}$, as shown in Table 3 . The range of temperatures derives from the difference in the monomeric units used in each proteinoid. When using phenylalanine, as in $\mathrm{P}(\mathrm{EF})$ and $\mathrm{P}(\mathrm{EIF})$, the resulted proteinoid gains rigidity in the overall structure, due to the aromatic rings which allow pi-stacking. Moreover, when SMO is encapsulated, the decomposition temperatures increases (by about

\begin{tabular}{|c|c|c|c|c|c|c|}
\hline Proteinoida & $M n(k D a)^{b}$ & $M w(k D a)^{b}$ & $M p(k D a)^{b}$ & $\mathrm{PDI}^{\mathrm{c}}$ & 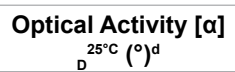 & $\begin{array}{l}\text { Carboxyl groups } \\
(\mathrm{mmol} / \mathrm{g})^{\mathrm{e}}\end{array}$ \\
\hline$P(E I)$ & 66 & 68 & 55 & 1.04 & -1.7 & 97 \\
\hline$P(E I F)$ & 57 & 61 & 44 & 1.07 & 4.1 & 85 \\
\hline$P(E F)$ & 53 & 57 & 42 & 1.07 & 1.2 & 97 \\
\hline
\end{tabular}

aThe proteinoids were prepared at $180^{\circ} \mathrm{C}$ according to the experimental section; ${ }^{b}$ molecular weights were measured by $\mathrm{GPC}$, Mp is the molecular weight at the peak; ${ }^{\mathrm{C}} \mathrm{PD}$ is the polydispersity index, given by $\mathrm{Mw} / \mathrm{Mn}$; ${ }^{\mathrm{d}}$ specific optical rotation $\left(\mathrm{c}=1\right.$, in $\mathrm{H}_{2} \mathrm{O}$, at $25^{\circ} \mathrm{C}$ ); eeach experiment was performed 4 times, with an error of $0.5-2 \%$.

Table 2: Mw, Mn, Mp, PDI, optical activity and carboxyl groups of the various proteinoids.
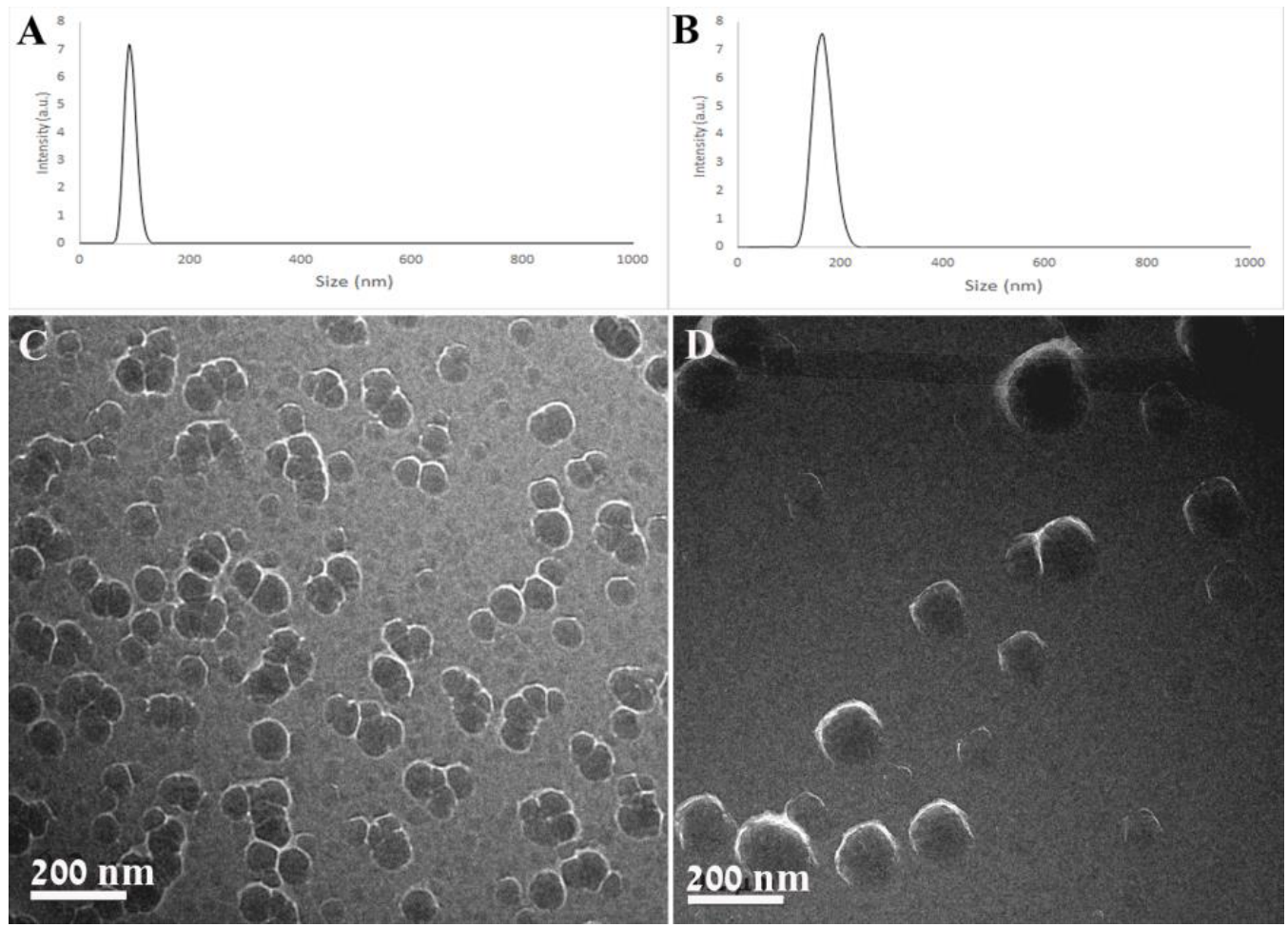

Figure 2: DLS histograms and cryo-TEM images of hollow $P(E I) N P s(A, C)$ and SMO-filled $P(E I) N P s(B, D)$, respectively. 


\begin{tabular}{|c|c|c|}
\hline Proteinoid NPs & Size $(\mathrm{nm})^{\mathrm{c}}$ & $\operatorname{Tdec}\left({ }^{\circ} \mathrm{C}\right)^{\circ}$ \\
\hline$P(E I) N P s^{a}$ & $89.7 \pm 6$ & 290 \\
\hline SMO-filled P(EI) NPs ${ }^{b}$ & $166.1 \pm 20$ & 310 \\
\hline$P(E I F) N P s^{a}$ & $125.9 \pm 12$ & 300 \\
\hline SMO-filled P(EIF) NPs ${ }^{b}$ & $202.8 \pm 23$ & 315 \\
\hline$P(E F) N P s^{a}$ & $119.0 \pm 16$ & 300 \\
\hline SMO-filled P(EF) NPs ${ }^{b}$ & $356.0 \pm 32$ & 310 \\
\hline
\end{tabular}

aHollow proteinoid NPs prepared by self-assembly; 'SMO-filled proteinoid NPs with $10 \%$ encapsulated SMO; 'size and size distribution measured by Nanophox; ${ }^{\mathrm{d}} \mathrm{Tdec}$ (temperature of decomposition) was measured by TGA/DSC. Each experiment was performed 3 times.

Table 3: Size and decomposition temperature of the different NPs.

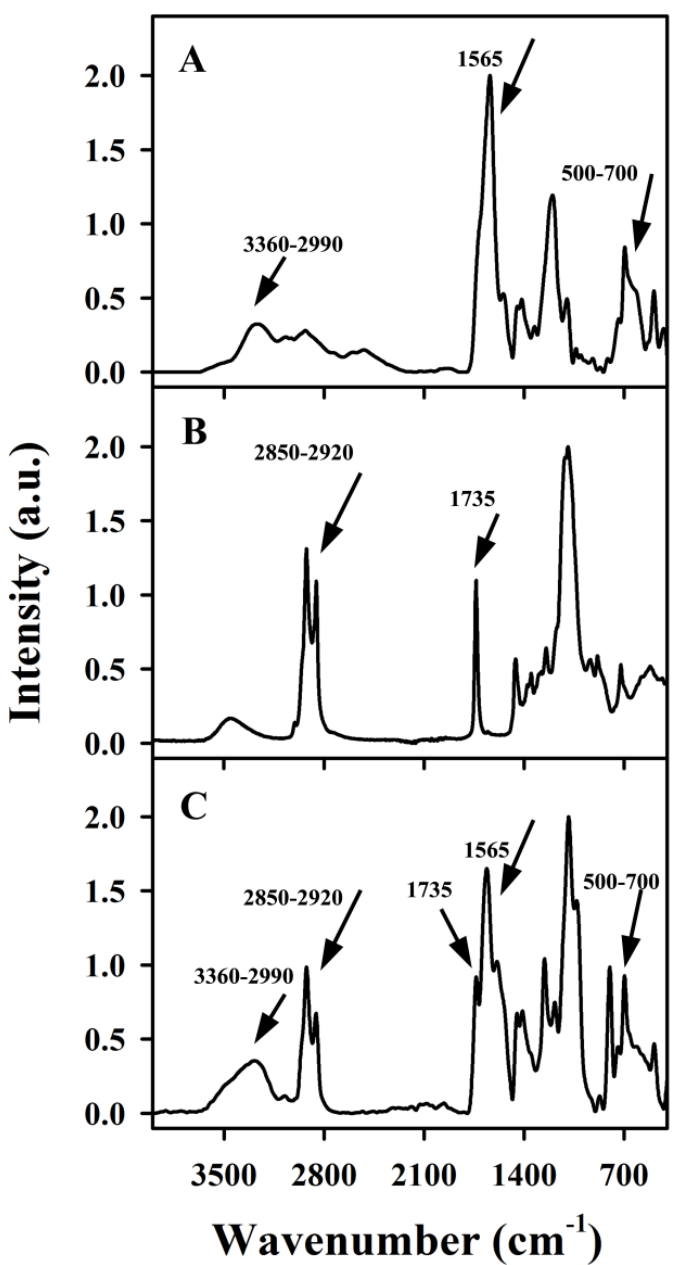

Figure 3: FTIR spectrum of hollow P(EF) NPs (A), SMO (B) and SMO-filled $P(E F)$ NPs $(C)$.

$10^{\circ} \mathrm{C}$ ), probably due to the hydrophobic interactions between the inner part of the proteinoid particles and the SMO.

The proteinoid particles were also characterized by infrared spectroscopy, as shown in Figure 3. Figure 3A exhibits the spectrum of $\mathrm{P}(\mathrm{EF}) \mathrm{NPs}$ as a typical spectrum for all the proteinoid particles, which show the same characteristic peaks; NH stretching at 3360-2990 $\mathrm{cm}^{-1}$, amide CO stretching at $1565 \mathrm{~cm}^{-1}$, and CO bending at $500-700 \mathrm{~cm}^{-1}$.

Figure $3 \mathrm{~B}$ exhibits the FTIR spectrum of free SMO, including characteristic peaks of $\mathrm{C}=\mathrm{O}$ stretching at $1735 \mathrm{~cm}^{-1}, \mathrm{C}=\mathrm{C}$ stretching at $722 \mathrm{~cm}^{-1}$ and $1350-1450 \mathrm{~cm}^{-1}$, and $\mathrm{CH}$ at $2850-2920 \mathrm{~cm}^{-1}$.
Figure 3C exhibits the spectrum of SMO-filled P(EF) NPs. It can be seen that the SMO-filled NPs possess a combination of the characteristic peaks of SMO and $\mathrm{P}(\mathrm{EF})$. Hence, it can be assumed that the encapsulation of SMO was successful.

\section{Coated PP film characterization}

PP films were coated by the proteinoids and proteinoid NPs and measured for their surface roughness and contact angle. Results are shown in Table 4 . For the roughness measurements, it is clearly shown that coating by proteinoids or proteinoid NPs improves the smoothness of the film. PP films that were treated by air corona, showed the highest surface roughness of $4.7 \mathrm{~nm}$. The high roughness is caused by the collisions of oxygen ions with the film that create pores in the PP film. Coating the PP film with the film former A131-X itself, did not improve the roughness, as it decreased insignificantly to $3.8 \pm 0.9 \mathrm{~nm}$. However, PP films after coating with the proteinoids or proteinoid NPs display roughness in the range of $0.7-1.5 \mathrm{~nm}$. The surface roughness significantly decreases and the film becomes smoother compared to the uncoated PP. The proteinoids and proteinoid NPs fill the film pores and form a smoother surface. It is clearly seen in Figure 4 that the rough surface of uncoated PP film (Figure 4A) compared to the smooth topographic surface of $\mathrm{P}(\mathrm{EF})$-coated $\mathrm{PP}$ (Figure $4 \mathrm{~B}$ ) and $\mathrm{P}(\mathrm{EI})$-coated $\mathrm{PP}$ (Figure 4C) with roughness of $4.7 \pm 0.2,0.7 \pm 0.1$ and $1.2 \pm 0.6$ $\mathrm{nm}$, respectively. The roughness of $\mathrm{P}(\mathrm{EI})$ is lower than $\mathrm{P}(\mathrm{EF})$, due to the incorporation of isoleucine in the proteinoid backbone which improves the hydrophobic interaction between the $\mathrm{P}(\mathrm{EI})$ and the PP films. The similarity in the chemical structure between the isoleucine side chain group and the PP films form better compatibility than a phenylalanine side chain. Phenylalanine consists of an aromatic ring side chain that prevents the interaction and the compatibility between the $\mathrm{P}(\mathrm{EF})$ and the PP films.

The proteinoids and proteinoid particles coatings also decrease the contact angle of the PP films to the required values for anti-fogging properties, shown in Table 4. The low contact angle degrees indicate the hydrophilicity of the coated films, hence achieving a good wetting character. Air corona-treated PP films possess a low water contact angle of $29^{\circ}$, compared to untreated PP with a contact angle of $90^{\circ}$. Nevertheless, fog accumulates on the air corona-treated PP film surface during 5 minutes of the hot fog test, as shown in Table 5. This can be probably explained by the high surface roughness of the uncoated PP film. As mentioned above, in order to achieve a good anti-fog coating, both surface roughness and low contact angle are important.

\begin{tabular}{|l|c|c|}
\hline Coating type $^{\mathbf{a}}$ & Roughness $_{\mathbf{( n m}} \mathbf{b}^{\mathbf{b}}$ & Contact angle $^{\left({ }^{\circ}\right)^{\mathbf{b}}}$ \\
\hline Uncoated PP & $4.7 \pm 0.2$ & $29 \pm 1$ \\
\hline A131-X & $3.8 \pm 0.9$ & $70 \pm 2$ \\
\hline $\mathrm{P}(\mathrm{EI})$ & $0.7 \pm 0.1$ & $7 \pm 0.7$ \\
\hline $\mathrm{P}(\mathrm{EI}) \mathrm{NPs}$ & $0.8 \pm 0.3$ & $12 \pm 1$ \\
\hline SMO-filled P(EI) NPs & $1.2 \pm 0.3$ & $30 \pm 0.5$ \\
\hline $\mathrm{P}(\mathrm{EIF})$ & $1.1 \pm 0.5$ & $6.5 \pm 0.7$ \\
\hline $\mathrm{P}(\mathrm{EIF}) \mathrm{NPs}$ & $1.4 \pm 0.6$ & $11 \pm 2$ \\
\hline SMO-filled P(EIF) NPs & $1.3 \pm 0.6$ & $40.5 \pm 1$ \\
\hline $\mathrm{P}(\mathrm{EF})$ & $1.2 \pm 0.6$ & $9 \pm 1$ \\
\hline $\mathrm{P}(\mathrm{EF})$ NPs & $1.5 \pm 0.6$ & $14.5 \pm 1$ \\
\hline SMO-filled P(EF) NPs & $1.5 \pm 0.7$ & $24 \pm 1.5$ \\
\hline
\end{tabular}

aAll samples refer to air corona-treated PP films (A4 $30 \mu \mathrm{m}$ ) coated with film former A131-X, proteinoid or proteinoid particles as specified, at $5 \% \mathrm{w} / \mathrm{w}$ to $\mathrm{A} 131-\mathrm{X}$, as described in the experimental part; beach result represents an average of three measurements at three different areas of the film.

Table 4: Surface roughness of the various films. 


\begin{tabular}{|c|c|c|c|c|c|c|}
\hline \multirow[t]{2}{*}{ Coating type $^{a}$} & \multicolumn{3}{|c|}{ Hot test Time (min) } & \multicolumn{3}{|c|}{ Cold test Time (min) } \\
\hline & 5 & 60 & 180 & 5 & 60 & 180 \\
\hline Uncoated PP & $\mathrm{D}$ & $\mathrm{D}$ & $\mathrm{D}$ & $\mathrm{D}$ & $\mathrm{D}$ & $\mathrm{D}$ \\
\hline A131-X & $\mathrm{D}$ & $\mathrm{D}$ & $\mathrm{D}$ & $\mathrm{D}$ & $\mathrm{D}$ & $\mathrm{D}$ \\
\hline$P(E I)$ & $A$ & A & A & B & A & $A$ \\
\hline$P(E I) N P s$ & $\mathrm{~B}$ & A & A & C & A & $A$ \\
\hline SMO-filled P(EI) NPs & $\mathrm{D}$ & $\mathrm{B}$ & A & D & C & B \\
\hline$P(E I F)$ & $\mathrm{D}$ & $\mathrm{B}$ & A & D & C & B \\
\hline$P(E I F)$ NPs & $\mathrm{D}$ & $\mathrm{B}$ & A & $\mathrm{D}$ & C & B \\
\hline SMO-filled P(EIF) NPs & $\mathrm{D}$ & $\mathrm{B}$ & $A$ & $\mathrm{D}$ & $\mathrm{C}$ & $B$ \\
\hline$P(E F)$ & $\mathrm{D}$ & A & A & $\mathrm{D}$ & $\mathrm{D}$ & $B$ \\
\hline$P(E F) N P s$ & $\mathrm{D}$ & $\mathrm{B}$ & A & $\mathrm{D}$ & $\mathrm{D}$ & $B$ \\
\hline SMO-filled P(EF) NPs & $\mathrm{D}$ & B & $A$ & $D$ & $D$ & $B$ \\
\hline
\end{tabular}

${ }^{a}$ All samples refer to PP films coated with film former A131-X, proteinoid or proteinoid particles as specified, at $5 \% \mathrm{w} / \mathrm{w}$ to $\mathrm{A} 131-\mathrm{X}$.

Table 5: Hot and cold fog test over $3 \mathrm{~h}$.
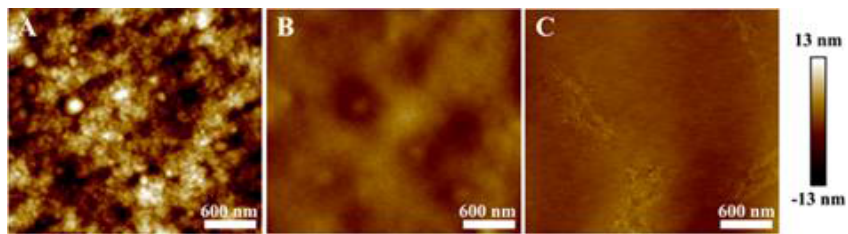

Figure 4: AFM images of PP (A), PP film coated with $P(E F)(B)$ and PP film coated with $\mathrm{P}(\mathrm{EI})(\mathrm{C})$
A

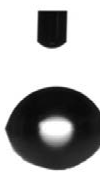

B

Figure 5: Contact angle images of $P P$ film coated with film former $A 131-X(A)$ $P P$ film coated with SMO-filled P(EI) NPs (B), PP film coated with $P(E I) N P s(C)$ and PP film coated with $\mathrm{P}(\mathrm{EI})(\mathrm{D})$

A131-X coating increased the contact angle to $70 \pm 2^{\circ}$, thus affecting the hydrophilicity and the anti-fog character of the film. However, it was used as a film former with the proteinoids and proteinoid NPs and gave low contact angles. Overall, using the proteinoids in their crude form yielded better results than the NPs. Additionally, it can be seen that the encapsulation of SMO within proteinoid particles did not improve the roughness significantly, and affected the contact angle, compared to proteinoid-coated PP. Figure 5 shows the contact angles of A131-X coated, SMO-filled P(EI) NPs, hollow P(EI) NPs and P(EI) in the form of a free proteinoid. As seen, the proteinoid particles give a higher contact angle compared to the proteinoids in the free form.

Figure 6 illustrates the visual difference between uncoated and proteinoid coated PP films over time. As seen, the water spread into a continuous and uniform layer only on the coated film (B, D and F). Nevertheless, on uncoated PP films the water form ball-shaped droplets on the surface (A, C and E). The coating changes the interfacial tension between the water and the PP surface and enables strong connectivity between the film and the water droplets.

The haze and gloss of coated and uncoated PP films (Table 6). PP film after coating by proteinoid polymer or proteinoid particle exhibit similar values of haze and gloss as uncoated PP film, all in the range of 2.002.70 and 80.9-91.0, respectively. Hence, the coatings in all forms may be used for many applications requiring relatively non-hazy and glossy films.

\section{Optical properties}

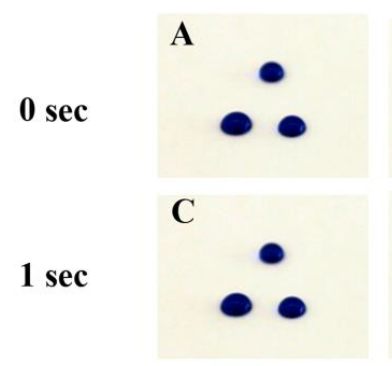

B

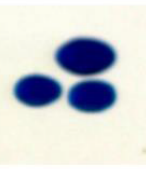

D

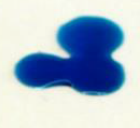

E

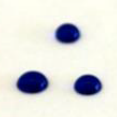

$\mathbf{F}$

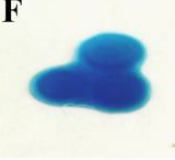

Coated
Figure 6: Water droplets on an uncoated PP film at different time periods ( $A$, $C$ and $E$ ), and on a coated PP films ( $B, D$ and $F$ ). The presented coating is $\mathrm{P}(\mathrm{EI})$. Water droplets were dyed with brilliant blue FCF to show contrast (similar results were obtained in the absence of dye).

\section{Fog test}

The coated films were tested in hot fog and cold fog as described in the experimental section, all the results were over $3 \mathrm{~h}$ of heating in $60^{\circ} \mathrm{C}$ and cooling in $4^{\circ} \mathrm{C}$, Table 5 . Figure 7 shows the range of optical visibility through the films, ranked as A-D.

Figure 8 shows the Mayer rod coating setup for coating on plastic films. The PP plastic film is put on a flat surface and the Mayer rod is pulled over the mixture of proteinoid or proteinoid NPs with A131-X in water, yielding a uniform layer with thicknesses of $6 \mu \mathrm{m}$ (Figure 8A).

Uncoated PP films show poor visibility, ranked as D over $3 \mathrm{~h}$, with no change (Figure 8B and 8D). Coating the films with A131-X does not improve the visibility during the hot or cold fog tests. However, when coated by the proteinoids or proteinoid particles in A131-X the visibility improves to rank $A$ in hot fog and to rank $A / B$ in cold fog. The film coated with $\mathrm{P}(\mathrm{EI})$ shows the best optical visibility, rank A, received within $5 \mathrm{~min}$ and remaining the same over $3 \mathrm{~h}$ in the hot fog test (Figure $8 \mathrm{C}$ and $8 \mathrm{E})$. Coating with $\mathrm{P}(\mathrm{EF})$ shows rank A only after $60 \mathrm{~min}$. The difference in the results may be due to the chemical structure of the proteinoids. As mentioned earlier, $\mathrm{P}(\mathrm{EI})$ contains isoleucine which is similar in the chemical structure to the PP film structure. The similarity in the chemical structure leads to a better compatibility between the film and the proteinoid mixtures. An improvement can also be seen 
Citation: Sason E, Kolitz-Domb M, Cohen S, Grinberg I, Margel S (2017) Engineering of New Proteinoids and Proteinoid Nanoparticles of Narrow Size Distribution for Anti-fog Applications. J Nanomed Nanotechnol 8: 473. doi: 10.4172/2157-7439.1000473

Page 7 of 9

\begin{tabular}{|l|c|c|}
\hline Coating type $^{\mathbf{a}}$ & Haze $^{\mathbf{b}}$ & Gloss $^{\mathbf{b}}$ \\
\hline Uncoated PP & $2.70 \pm 0.10$ & $82.0 \pm 1.6$ \\
\hline A131-X & $2.36 \pm 0.13$ & $80.9 \pm 7.2$ \\
\hline $\mathrm{P}(\mathrm{EI})$ & $2.29 \pm 0.17$ & $88.5 \pm 1.4$ \\
\hline $\mathrm{P}(\mathrm{EI}) \mathrm{NPs}$ & $2.00 \pm 0.11$ & $84.3 \pm 2.0$ \\
\hline SMO-filled P(EI) NPs & $2.16 \pm 0.02$ & $86.6 \pm 1.6$ \\
\hline $\mathrm{P}(\mathrm{EIF})$ & $2.30 \pm 0.15$ & $88.4 \pm 2.6$ \\
\hline $\mathrm{P}(\mathrm{EIF}) \mathrm{NPs}$ & $2.22 \pm 0.11$ & $91.0 \pm 1.2$ \\
\hline SMO-filled P(EIF) NPs & $2.53 \pm 0.14$ & $85.8 \pm 1.2$ \\
\hline $\mathrm{P}(\mathrm{EF})$ & $2.54 \pm 0.18$ & $90.9 \pm 0.8$ \\
\hline $\mathrm{P}(\mathrm{EF})$ NPs & $2.38 \pm 0.14$ & $86.6 \pm 1.6$ \\
\hline SMO-filled P(EF) NPs & $2.31 \pm 0.26$ & $84.1 \pm 8.6$ \\
\hline
\end{tabular}

aAll samples refer to PP films coated with film former A131-X, proteinoid or proteinoid particles as specified, at $5 \% \mathrm{w} / \mathrm{w}$ to $\mathrm{A} 131-\mathrm{X}$; beach result represents an average of three measurements at three different areas of the film.

Table 6: Haze and gloss of the various films.

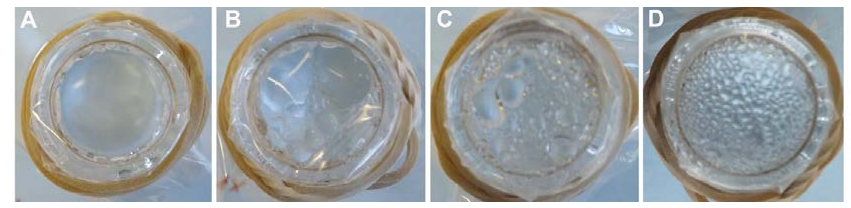

Figure 7: Optical visibility ranking: (A) transparent continuous layer of water excellent optical performance; (B) large water drops on some parts of the surface allowing partial light transition; (C) medium water drops on most of the surface allowing partial light transition; (D) small water drops on the whole surface, causing very poor visibility.

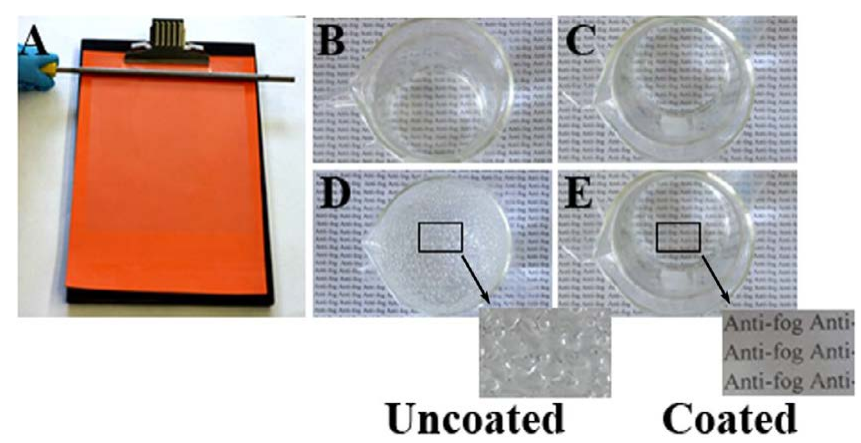

Figure 8: Mayer rod coating setup for coating on plastic films. The PP plastic film is put on a flat surface and the Mayer rod is pulled over the mixture of proteinoid or proteinoid NPs with A131-X in water, which leaves a uniform layer with thicknesses of $6 \mu \mathrm{m}(A)$, images of uncoated PP film (B) and $P(E I)$-coated $P P$ film (C) at time zero, uncoated PP film (D) and P(EI)-coated PP film (E) after $3 \mathrm{~h}$ in $60^{\circ} \mathrm{C}$.

when coating with $\mathrm{P}(\mathrm{EI})$ and $\mathrm{P}(\mathrm{EF})$ hollow and SMO-filled particles, but over more time (in hot fog, 5-60 min to rank $\mathrm{B}$ and $3 \mathrm{~h}$ to rank $\mathrm{A}$ and in cold fog $3 \mathrm{~h}$ to rank B). The stability of the coatings was examined by hot fog tests of the films after storage in a laboratory cupboard for 12 months. The films showed unchanged results as the results achieved after $3 \mathrm{~h}$ of the test, as shown in the table.

Spray-coated glass was also tested in comparison to uncoated glass, by the hot fog test as described in the experimental section, the results were over $3 \mathrm{~h}$ of heating in $60^{\circ} \mathrm{C}$. Figure 9 shows the clear results achieved within the first $5 \mathrm{~min}$ of the test, where the left glass presented is uncoated and the fog rank is D, opposed to the right glass which is coated and ranked $\mathrm{A}$. The difference in fog accumulation on the glass surfaces is well expressed by the visibility of the paper placed under the coated and uncoated glass.

\section{Cellular cytotoxicity of the proteinoids and proteinoid particles by $\mathrm{LDH}$ assay}

In order to revoke the cell toxicity of the proteinoids and proteinoid particles, in vitro cytotoxicity was tested using osteosarcoma U-2 OS cell lines. Cell cytotoxicity was assessed by measuring the release of cytoplasmic lactate dehydrogenase (LDH) into the cell culture supernatants. $\mathrm{LDH}$ is an intracellular enzyme which catalyzes the reversible oxidation of lactate to pyruvate. Since LDH is predominantly in the cytosol, the enzyme is released into the supernatant only upon cell damage or lysis [17].

When tested by the $\mathrm{LDH}$ quantitative assay, proteinoids and proteinoid particles dispersed in PBS $(1 \mathrm{mg} / \mathrm{mL}$ and $0.1 \mathrm{mg} / \mathrm{mL})$ had none, or minor cytotoxic effect on the osteosarcoma U-2 OS cells line compared to untreated (blank) cells, as demonstrated in Figure 10. Therefore, proteinoids and proteinoid particles can be suitable for various applications.

\section{Cellular cytotoxicity of the proteinoids and proteinoid particles by XTT assay}

The proteinoids and proteinoid particles were also tested by XTT

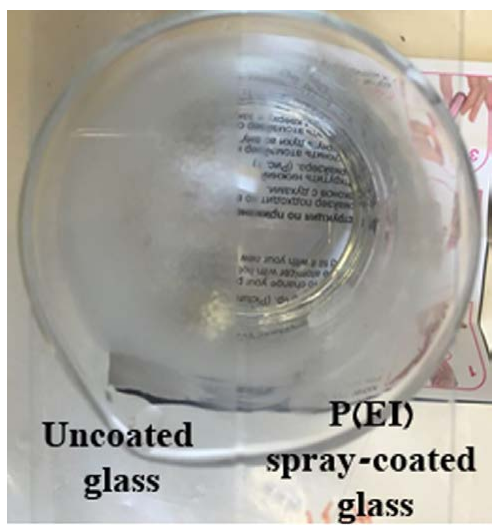

Figure 9: Hot fog test of uncoated (left) and $\mathrm{P}(\mathrm{EI})$ spray-coated glass (right).

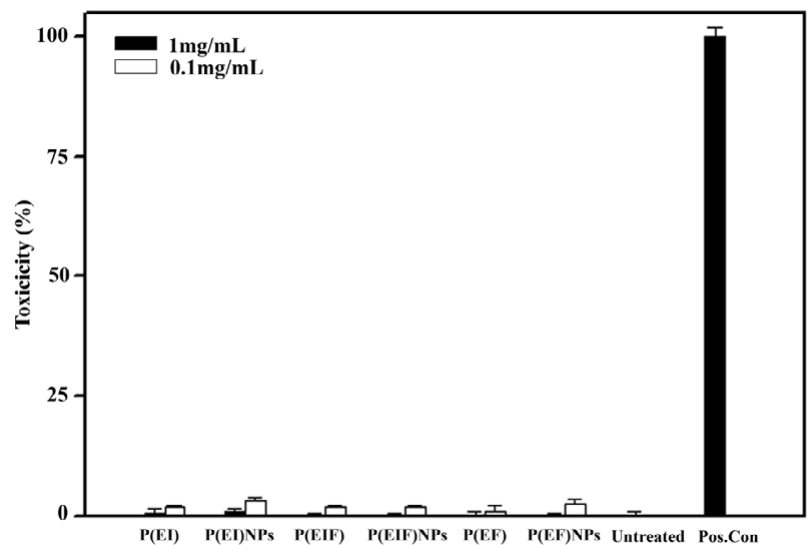

Figure 10: Cytotoxic effect of the proteinoids and proteinoids NPs on human osteosarcoma U-2 OS cells measured by the LDH assay. Cells $\left(3 \times 10^{5}\right)$ were incubated with the proteinoids and proteinoid particles dispersed in PBS (1 mg/ $\mathrm{mL}$ and $0.1 \mathrm{mg} / \mathrm{mL}$ ) according to the experimental section. Cells were incubated with Triton-x-100 $1 \%$ as positive control (100\% toxicity). In addition, cells were incubated with Triton-x-100 $1 \%$ and each one of the proteinoids to revoke any interaction. Untreated cells (negative control) were similarly incubated. Each bar represents mean \pm standard deviations of 4 separate samples. 


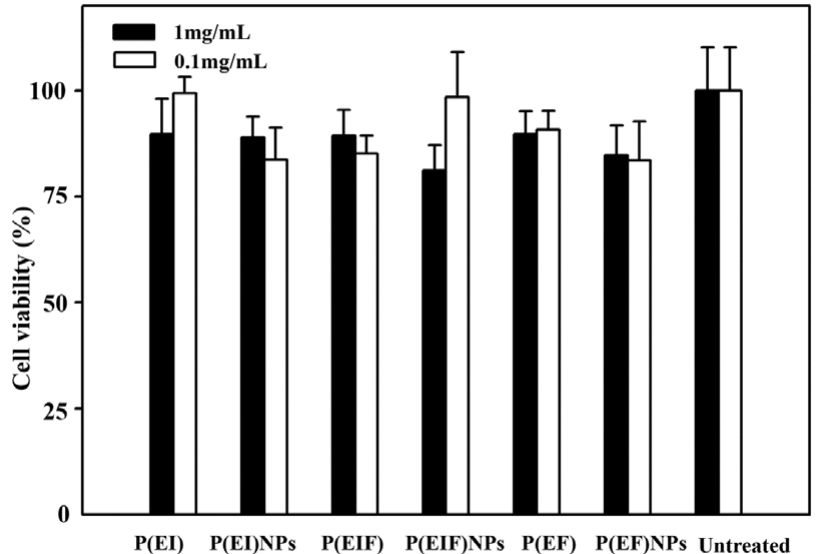

Figure 11: Cell viability levels of human osteosarcoma U-2 OS cells after exposure to the proteinoids and proteinoid particles measured by the XTT assay. Cells $\left(3 \times 10^{5}\right)$ were incubated for $72 \mathrm{~h}$ with the proteinoids and proteinoid particles dispersed in PBS $(1 \mathrm{mg} / \mathrm{mL}$ and $0.1 \mathrm{mg} / \mathrm{mL})$ according to the experimental section. Untreated cells (positive control) were similarly incubated. Each bar represents mean \pm standard deviations of 4 separate samples.

assay for in vitro cell viability following exposure to the proteinoids and particles. In order to determine the number of viable cells the XTT assay uses 2,3-Bis-(2-methoxy-4-nitro-5-sulfophenyl)-2H-tetrazolium-5 carboxanilide salt (XTT) [22]. Only in living cells, mitochondria are capable to reduce XTT to form orange colored water-soluble dye, which its concentration is proportional to the number of living cells and can be calculated.

Human osteosarcoma U-2 OS cells were exposed to different concentrations $(1 \mathrm{mg} / \mathrm{mL}$ and $0.1 \mathrm{mg} / \mathrm{mL})$ of the proteinoids and proteinoid particles. Figure 11 exhibits the cell viability levels of the cells post treatment. Treatment of U-2 OS cells with $1 \mathrm{mg} / \mathrm{mL}$ and 0.1 $\mathrm{mg} / \mathrm{mL}$ in PBS produced a minor decrease in viability level (20\%) after $72 \mathrm{~h}$ when compared to untreated (blank) cells. Overall, the proteinoids and proteinoid nanoparticles can be considered as non-toxic and can be suitable for various applications.

\section{Summary and Conclusions}

In this study, a series of proteinoids containing Glu, Phe, and Ile was synthesized to form proteinoid and proteinoid NPs that encapsulate SMO for anti-fog application. The proteinoids, synthesized by thermal step-growth polymerization, were mostly uniform in size distribution with relatively high molecular weights. Following proteinoid synthesis, NPs were prepared by a self-assembly procedure and characterized for their size, thermal properties and FTIR spectrum. Then, coatings were made on air corona-treated PP films by mixture of a film former and proteinoids or nanoparticles. Surface roughness and contact angle of the films were characterized and shown hydrophilic coatings with static contact angle in the range of $7-40^{\circ}$ and a surface roughness in the range of 0.7-1.2 $\mathrm{nm}$. The optical characteristics of the coatings demonstrated good wettability and transparency for all the coatings, especially for the coated with proteinoid $\mathrm{P}(\mathrm{EI})$. However, coatings based on SMO-filled NPs did not achieve better anti-fogging abilities, and the proteinoid in its simple form achieved the best anti-fogging proficiency. The proteinoids and proteinoid NPs are non-toxic to human cells. Hence, proteinoids may potentially become useful as an anti-fog material for many devices, including biological or food-related applications. Further studies concerning the optimization of the proteinoid coatings for antifog application are ongoing in our laboratories.

\section{References}

1. Fox SW, Harada K (1995) Thermal synthesis of amino acids and the origin of life. Geochim Cosmochim Acta 59: 1213-1214.

2. Fox SW, Harada K, Waehneldt TV (1968) The thermal synthesis of neutral and basic proteinoids. Biochim Biophys Acta 160: 246-249.

3. Fox SW, Harada K (1960) The thermal copolymerization of amino acids common to protein. J Am Chem Soc 82: 3745-3751.

4. Kolitz-Domb M, Margel S (2014) Engineering of near infrared fluorescent proteinoid-poly (L-lactic acid) particles for in vivo colon cancer detection. Nanobiotech 12: 1-13.

5. Kolitz-Domb M, Grinberg I, Corem-salkmon E, Margel S (2014) Engineered narrow size distribution high molecular weight proteinoids, proteinoid-poly (L-lactic acid) copolymers and nano/micro-hollow particles for biomedical applications. Nanomed Nanotechnol 5: 2-10

6. Kolitz-Domb M, Corem-salkmon E, Grinberg I, Margel S (2014) Synthesis and characterization of bioactive conjugated near-infrared fluorescent proteinoidpoly (L-lactic acid) hollow nanoparticles for optical detection of colon cancer. Int J Nanomedicine 9: 5401-5453.

7. Kiel S, Kolitz-Domb M, Corem-Salkmon E, Margel S (2017) Engineered doxorubicin delivery system using proteinoid-poly (L-lactic acid) polymeric nanoparticles of narrow size distribution and high molecular weight for cancer treatment. Int J Nanotechnol Nanomedicine 2: 1-11.

8. Introzzi L, Fuentes-Alventosa JM, Cozzolino CA, Trabattoni S, Tavazzi S, et al. (2012) 'Wetting enhancer' pullulan coating for antifog packaging applications. ACS Appl Mater Interfaces 4: $\mid 3692-3700$.

9. Huang KT, Yeh SB, Huang CJ (2015) Surface modification for superhydrophilicity and underwater superoleophobicity: applications in antifog, underwater selfcleaning, and oil-Water separation. ACS Appl Mater Interfaces 7: 21021-21029.

10. Yuan Y, Liu R, Wang C, Luo J, Liu X (2014) Synthesis of UV-curable acrylate polymer containing sulfonic groups for anti-fog coatings. Prog Org Coatings 77: 785-789.

11. Shlosman K, Suckeveriene RY, Rosen-Kligvasse J, Tchoudakov R, Zelikman $\mathrm{E}$, et al. (2014) Controlled migration of antifog additives from LLDPE compatibilized with LLDPE grafted maleic anhydride. Polym Adv Technol 25 1484-1491.

12. Kumar AM, Rao KP (1998) Preparation and characterization of pH-sensitive proteinoid microspheres for the oral delivery of methotrexate. Biomaterials 19 725-732.

13. Syren RM, Sanjur A, Fox SW (1985) Proteinoid microspheres more stable in hot than in cold water. Biosystems 17: 275-280.

14. Cohen S, Haham H, Pellach M, Margel S (2016) Design of UV-absorbing polypropylene films with polymeric benzotriaziole based nano- and microparticle coatings. ACS Appl Mater Interfaces 9: 868-875

15. Nanowire S, Hu L, Kim HS, Lee JY, Peumans P, et al. (2010) Scalable coating and properties of transparent, flexible, silver nanowire electrodes. ACS Nano 4: $2955-2963$.

16. Howarter JA, Youngblood JP (2008) Self-cleaning and next generation anti-fog surfaces and coatings. Macromol Rapid Commun 29: 455-466.

17. Decker T, Lohmann-Matthes ML (1988) A quick and simple method for the quantitation of lactate dehydrogenase release in measurements of cellular cytotoxicity and tumor necrosis factor (TNF) activity. J Immunol Methods 115 61-69.

18. Izak-Nau E, Voetz M, Eiden S, Duschl A, Puntes VF (2013) Altered characteristics of silica nanoparticles in bovine and human serum: the importance of nanomaterial characterization prior to its toxicological evaluation. Part Fibre Toxicol 10: 56

19. Belostozky A, Grinberg I, Haham H, Margel S (2017) Nanomedicine \& nanotechnology engineering of new UV-blocking hollow proteinoid nanoparticles of narrow size distribution containing all-trans retinoic acid for biomedical applications. J Nanomed Nanotechnol 8: 1-9. 
Citation: Sason E, Kolitz-Domb M, Cohen S, Grinberg I, Margel S (2017) Engineering of New Proteinoids and Proteinoid Nanoparticles of Narrow Size Distribution for Anti-fog Applications. J Nanomed Nanotechnol 8: 473. doi: 10.4172/2157-7439.1000473

Page 9 of 9

20. Fox SW, Jungck JR, Nakashima T (1974) From proteinoid microsphere to contemporary cell-formation of internucleotide and peptide-bonds by proteinoid particles. Orig Life 5: 227-237.

21. Yuan Y, Liu R, Wang C, Luo J, Liu X (2014) Synthesis of UV-curable acrylate polymer containing sulfonic groups for anti-fog coatings. Prog Org Coat 4 785-789.

22. Lanone S, Rogerieux F, Geys J, Dupont A, Maillot-Marechal E, et al. (2009) Comparative toxicity of 24 manufactured nanoparticles in human alveolar epithelial and macrophage cell lines. Part Fibre Toxicol 6: 14. 